EPJ Web of Conferences 31, 00035 (2012)

DOI: $10.1051 /$ epjconf/20123100035

(C) Owned by the authors, published by EDP Sciences - SIF, 2012

\title{
FARCOS, a new array for femtoscopy and correlation spectroscopy
}

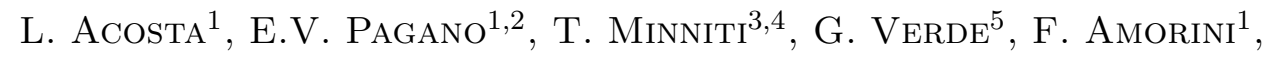
A. Anzalone ${ }^{1}$, L. Auditore ${ }^{3,4}$, M. Buscemi ${ }^{1,2}$, G. Cardella ${ }^{5}$, A. $\mathrm{ChBihi}^{6}$, E. De Filippo ${ }^{5}$, L. Francalanza ${ }^{1,2}$, E. Geraci $^{2,5}$, S. Gianì ${ }^{1,2}$, C. Guazzoni ${ }^{7,8}$, E. La Guidara ${ }^{5,9}$, G. Lanzalone ${ }^{1,10}$, I. LOMBARdo ${ }^{11,12}$, S. Lo Nigro ${ }^{2,5}$, D. LORIA ${ }^{3,4}$, C. MAIOLINO ${ }^{1}$, I. Martel ${ }^{13}$, A. Pagano ${ }^{5}$, M. Papa ${ }^{5}$, S. Pirrone ${ }^{5}$, G. Politi ${ }^{2,5}$, F. Porto ${ }^{1,2}$, F. Rizzo ${ }^{1,2}$, P. Russotto ${ }^{1,2}$, A.M. SÁnchez-Bentez ${ }^{13}$, J.A. Duenas ${ }^{13}$, R. Berjillos ${ }^{13}$, S. Santoro ${ }^{3,4}$, A. Trifirò ${ }^{3,4}$, M. Trimachi ${ }^{3,4}$, M. Venhart ${ }^{14}$, M. VeSelsky ${ }^{14}$ and M. Vigilante ${ }^{11,12}$

${ }^{1}$ INFN-LNS, Catania, Italy

${ }^{2}$ Dipartimento di Fisica e Astronomia, Università degli Studi di Catania, Italy

${ }^{3}$ INFN, Gruppo Collegato di Messina, Italy

${ }^{4}$ Dipartimento di Fisica Università degli Studi di Messina, Italy

${ }^{5}$ INFN, Sezione di Catania, Italy

${ }^{6}$ GANIL, CEA-IN2P3-CNRS, Caen, France

${ }^{7}$ INFN, Sezione di Milano, Italy

${ }^{8}$ Dipartimento di Fisica, Università degli Studi di Milano, Italy

${ }^{9}$ CSFNSM, Catania, Italy

${ }^{10}$ Università Kore, Enna, Italy

${ }^{11}$ INFN, Sezione di Napoli, Italy

${ }^{12}$ Dipartimento di Fisica, Università di Napoli, Italy

${ }^{13}$ Universidad de Huelva, Spain

${ }^{14}$ Slovak Academy of Sciences, Bratislava, Slovakia

This is an Open Access article distributed under the terms of the Creative Commons Attribution License 2.0, which permits unrestricted use, distribution, and reproduction in any medium, provided the original work is properly cited. 


\begin{abstract}
Correlations between two or more particles can be used as a tool to explore the space-time features of nuclear reactions as well as spectroscopic properties of produced unbound clusters. In order to have new options to study the mentioned correlations, FARCOS (Femtoscope ARray for COrrelations and Spectroscopy) has been conceived as a compact high resolution array, composed of square telescopes. In this work the main features of FARCOS array as well as part of the physics cases are described.
\end{abstract}

\title{
1 Introduction
}

When correlations between different particles emitted during a collision are studied, it is possible to find information about the space-time properties and quantitative understanding of reaction dynamics. Such studies depend on the details of the nuclear interaction and the equation of state (EoS) of nuclear matter. To reach highly precise experimental results, needed to study such interactions, detectors capable of detecting all reaction products on an event-by-event basis and measure their reciprocal correlations are mandatory $[1,2]$. Different observables need to be measured over a large solid angle coverage (making sure a good characterization of the collision event) with high energy and angular resolution (to register the kinetic energy and to obtain the momentum vectors and their correlations). Recent implementation of pulse-shape identification techniques gives promising expectative to provide unique capabilities $[3,4]$ that will allow studying nuclear dynamics even at low energies. The FARCOS detector (Femtoscope ARray for COrrelations and Spectroscopy) has been designed as a compact high resolution array, constituted by telescopes, each composed of square clusters of Double-Sided Silicon Strip Detectors (DSSSD) and CsI(Tl) crystals. The array will address topics covering both nuclear dynamics and spectroscopy with stable and radioactive beams.

By means of high resolution imaging techniques, providing sort of "spacetime" snapshots of particle and complex fragments emitting sources, the new array will address the study of correlation measurements in nuclear dynamics $[5,6]$. Using the images produced is possible to probe the $\mathrm{N} / \mathrm{Z}$ degree of freedom in nuclear reactions and the sub-saturation density dependence of symmetry energy. The study of two- and multi-particle coincidence measurement from exotic fragments produced in collisions allows the quest of new resonances, providing important spectroscopic information of unbound states, such as spin and branching ratios with respect to new decay chan- 


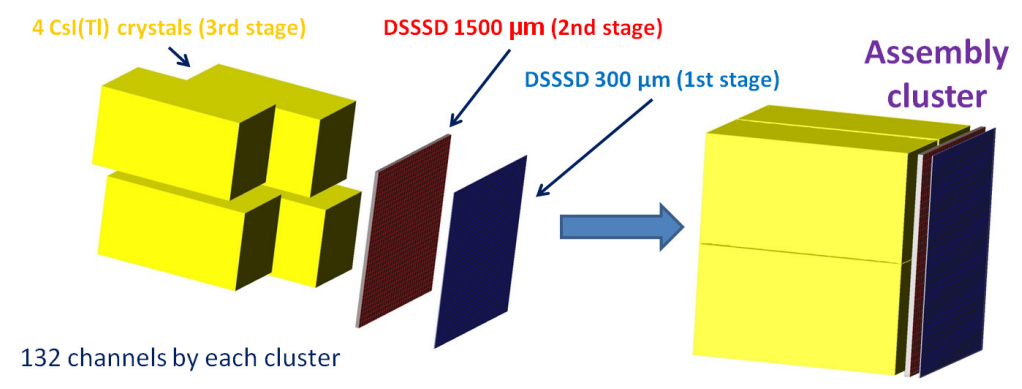

Figure 1: (Colour online). Different detection stages of the FARCOS array

nels and/or specific ones $[7,8]$. From this point of view it will be interesting to use the FARCOS array in coincidence with large $4 \pi$ detectors (such as CHIMERA and INDRA), in order to study prompt and sequential decays of exotic fragments such as ${ }^{10} \mathrm{C},{ }^{18} \mathrm{Ne}$, etc. The coupling of the FARCOS array to magnetic spectrometers and neutron detectors is also under study, with the aim of extending the physics reach of the project to spectroscopy studies conducted with stable and exotic beams. Along this work, the present status of the array, construction and design are described.

\section{Features of FARCOS array}

According to the topics exposed in the previous section, the FARCOS project presents different features. As it is well known, measurements over a wide dynamic range require using telescopes where thick $\mathrm{CsI}(\mathrm{Tl})$ crystals are included in combination with silicon detectors. The high stopping power of thick $\mathrm{CsI}(\mathrm{Tl})$ allows indeed stopping of high energy light particles, that are key for HBT studies in nuclear reactions. One of the most important features of FARCOS has to be the high spatial and energy resolution. In order to achieve such characteristic, FARCOS is composed of telescopes forming clusters, where each cluster contains double-sided silicon-strip detectors (DSSSD) of 300 and $1500 \mu \mathrm{m}$ of thickness, as first and second stages, respectively, followed by $4 \mathrm{CsI}(\mathrm{Tl})$ crystals in a "window shape" of $6 \times 32 \times 32 \mathrm{~mm}^{3}$, as a third stage. Each DSSSD is divided into 32 horizontal and 32 vertical strips, with which it is possible to generate individual pixels of $2 \times 2 \mathrm{~mm}^{2}$. The total detection area of the silicon detectors is $64 \times 64 \mathrm{~mm}^{2}$, adapted to cover the total area of the $4 \mathrm{CsI}(\mathrm{Tl})$ crystals placed behind. The scheme of 


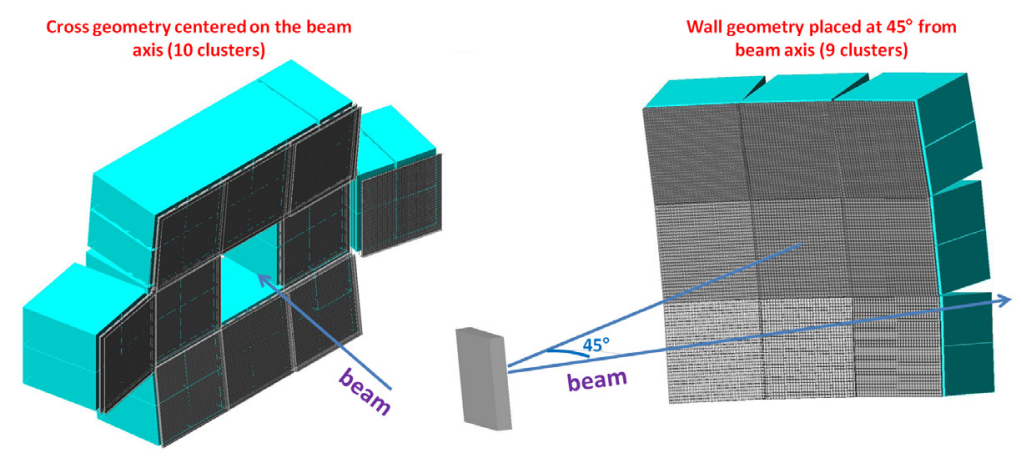

Figure 2: (Colour online). On the left it is show a configuration of FARCOS "in cross", composed by 10 clusters, whereas on the right it can be seen a geometry in wall, placed at $600 \mathrm{~mm}$ from target and with the center of the array fixed at $45^{\circ}$ from beam axis. In both arrays, the geometry of each cluster follows the same radius with respect to the center of the target; it means that each detector is tangential to the imaginary sphere generated by a distance "target-array" previously fixed.

the different stages of one FARCOS cluster is shown on Fig. 1. The current features of the different stages of the FARCOS array will allow to achieve a high resolution not only in energy measurements but also in the detection polar and azimuthal angles, $\theta$ and $\phi$. The basic idea is the capability of building different geometries grouping the FARCOS clusters in various configurations. Two of these possible geometries, using 9 and 10 clusters, can be schematically observed in Fig. 2, respectively. The requirements of each experiment will drive the geometry to be constructed.

\section{Detectors of FARCOS and present status}

As it was mentioned before, FARCOS is composed of $\mathrm{CsI}(\mathrm{Tl})$ crystals and DSSSD detectors. The first ones are highly homogeneous CsI(Tl) crystals made by SCIONIX [9]. Each crystal is wrapped with $0.12 \mathrm{~mm}$ thick white reflector including 50 micron of aluminized Mylar. The entrance window is composed by $2 \mu \mathrm{m}$ thick aluminized Mylar with a density of $0.29 \mathrm{~g} / \mathrm{cm}^{2}$. The $\mathrm{Tl}$ concentration is of the order of 1200 to $1500 \mathrm{ppm}$; higher concentrations are not recommended since it can produce a higher self absorption. The output light is read by a Hamamatsu 18x18 mm PIN diode S3204-08, attached to the rear face of each crystal. In the case of DSSSD, they were made by MICRON Semiconductor [10], based on a standard design (BB7) 


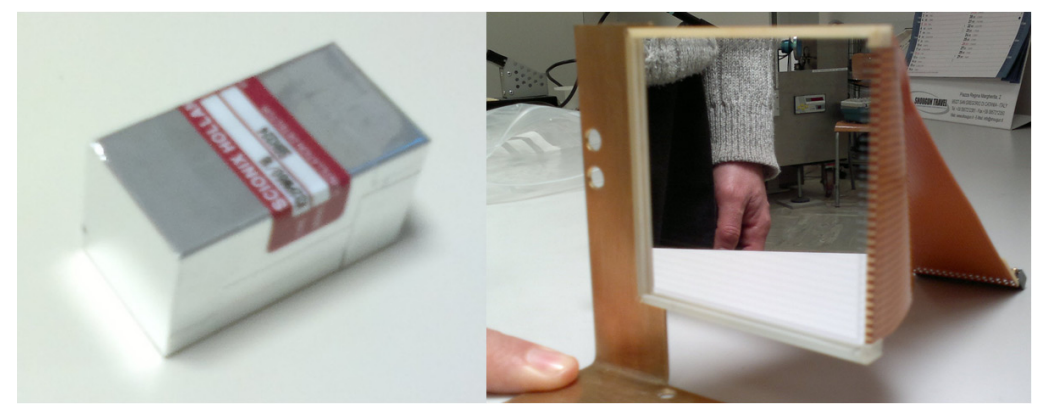

Figure 3: (Colour online). Different types of FARCOS detectors: On the left a typical FARCOS CsI $(\mathrm{Tl})$ crystal. Right panel shows a typical DSSSD of the FARCOS array.

with a minimum area PCB frame (4 mm of length). Each detector has two kapton cables welded to two perpendicular sides from the detector, for BIAS and readout issues. Each cable manages the signal of 32 strips from junction and ohmic faces of each detector respectively. In addition, a common ground and a BIAS guard-ring can also be managed with kapton cables. Each of these detectors can be held using $1 \mathrm{~mm}$ holes placed on 4 corners of its PCB. Pictures of CsI(Tl) crystals and DSSSD of FARCOS are depicted on Fig. 3.

In order to obtain the modularity and flexibility required for FARCOS, it is important to find solutions for the ideal mechanical structure to hold the whole system. Along this direction, it has been proposed to use an independent mechanics for each cluster, including in each of them, a special piece to assembly one with each other. This way, each cluster of FARCOS is thought to be mounted and dismounted without disturbing the other ones. Preliminary designs for the mechanics of FARCOS are shown in Fig. 3. Such mechanics is designed just to allow the minimum separation between each cluster. Thus, the active detection area of one cluster is separated by less than $10 \mathrm{~mm}$ from each other. This gap can be compensated by shifting properly neighboring clusters.

At present, $16 \mathrm{CsI}(\mathrm{Tl})$ crystals and $8 \mathrm{DSSSD}$ (4 of $300 \mu \mathrm{m}$-thick and 4 of $1500 \mu \mathrm{m}$-thick silicon) have been delivered and mounted. With them it is possible to construct the FARCOS demonstrator to test the capabilities of the future entire array. Current tests for silicon detectors using a triple-alpha source show preliminary optimal resolutions, making possible to observe even the secondary peaks in the energy spectra. These tests are car- 

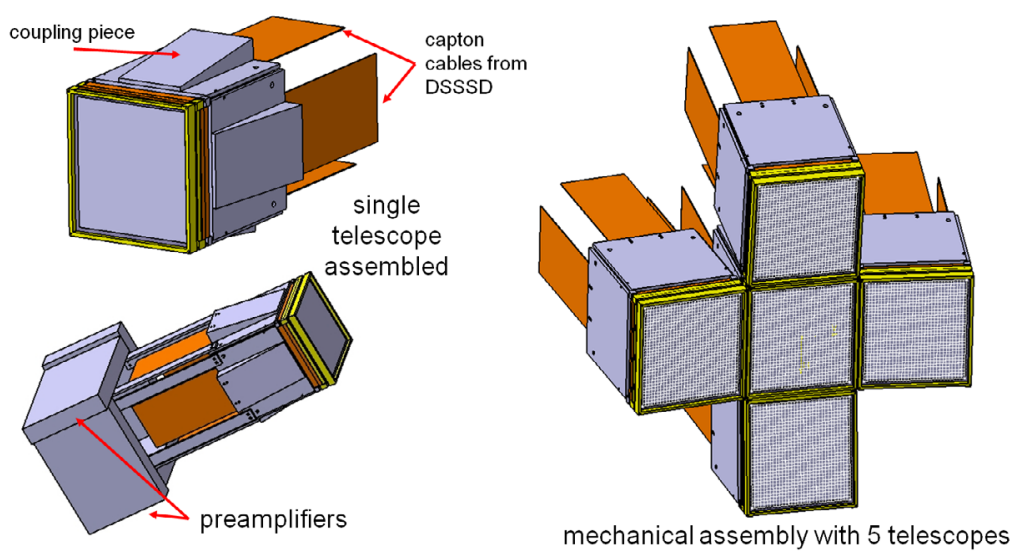

Figure 4: (Colour online). Preliminary mechanical structure for FARCOS array. In the left up-corner is show a scheme of one single cluster assembled, while in down corner is shown the same design including four preamplifier box, dedicated to the DSSSD's. On the right panel is depicted one realistic assembly composed by 5 clusters, all of them attached to the central one by means of a coupled metal piece.

ried out using commercial low-noise preamplifiers MPR-16 from Mesytec in combination with pulse-shaper discriminator N1568B modules from CAEN. The future designed electronics will improve the energy resolution and the low power consumption in order to extend towards very large scale integration (VLSI) solutions.

In the case of $\mathrm{CsI}(\mathrm{Tl})$, the test has to be depth, it is well known that the resolution can be non-homogenous, since light production can be nonuniform at the entrance of the crystal. For this reason, a special test is been carried out using a collimated monochromatic alpha source to scan the surface of the crystal, searching for notable differences in the output signals. These studies are important because knowledge of the impact position (provided by the front DSSSD) allows one to correct for non-uniformities, largely improving the energy resolution during the experiment. To perform the scanning, a single crystal is moved using high precision mechanical motors with respect to the alpha source. The preliminary results of this test show a negligible difference in the final resolution observed on the energy spectra generated. The fluctuation is around to $\pm 1 \%$ presenting a mean resolution of $4 \%$. Further studies of the $\mathrm{CsI}(\mathrm{Tl})$ crystals will be performed shortly. 


\section{Outlook}

In order to extend the characterization of $\mathrm{CsI}(\mathrm{Tl})$ detectors, testing the light output uniformity in deeper regions of the crystals [11-13], a beam test will be performed using the FARCOS demonstrator (3 clusters) at Laboratori Nazionali del Sud facility at Catania, Italy. In this test (expected to be carried out in summer 2012) beams of $\mathrm{H},{ }^{4} \mathrm{He}$ and ${ }^{12} \mathrm{C}$ at different energies will be used with different targets $\left({ }^{12} \mathrm{C},{ }^{2} \mathrm{H}\right.$ and $\left.\mathrm{H}\right)$, with the aim to study known reactions (such as $(\mathrm{p}, \mathrm{d}),(\mathrm{p}, \mathrm{t}),(\mathrm{d}, \mathrm{t}))$ as well as elastic collisions. The scattering particles at different angles and energies will be used to analyse the light-output uniformity of CsI crystals, due to the pixel granularity of the silicon detectors. During the test, different parameters related with the electronic devices can be simultaneously revised (such as comparison between different preamplifiers and silicon detector types). The results of this technical test will dictate the guidelines to construct the whole array. In order to reduce the power consumption and considering the high density of channels number, the possibility of implementing VLSI electronics will be also explored in a near future. Some examples of this kind of solutions can be consulted on existing arrays such as HYRA [14] or MUST2 [15].

\section{References}

[1] J. Pouthas et al., Nucl. Instr. and Meth. A 357 (1995) 418.

[2] A. Pagano et al., Nucl. Phys. A 681 (2001) 331c.

[3] A. Alderighi et al., IEEE Trans. on Nucl. Sci. 52 (2005) 1624.

[4] L. Bardelli et al., Nucl. Instr. and Meth. A 605 (2009) 353.

[5] G. Verde et al., Phys. Rev. C 65 (2002) 054609.

[6] G. Verde et al., Phys. Rev. C 67 (2003) 034606.

[7] W.P. Tan et al., Phys. Rev. C 69 (2004) 061304.

[8] F. Grenier et al., Nucl. Phys. A 811 (2008) 233.

[9] http://www.scionix.nl/.

[10] http://www.micronsemiconductor.co.uk/.

[11] S. Aiello et al., Nucl. Instr. and Meth. A 369 (1996) 50. 
EPJ Web of Conferences

[12] A. Wagner et al., Nucl. Instr. and Meth. A 456 (2001) 290.

[13] M.J. van Goethem et al., Nucl. Instr. and Meth. A 526 (2004) 526.

[14] M.S. Wallace et al., Nucl. Instr. and Meth. A 583 (2007) 302.

[15] E. Pollacco et al., Eur. Phys. J. A 25-s01 (2005) 287. 